\title{
Partitional Clustering-Hybridized Neuro-Fuzzy Classification Evolved through Parallel Evolutionary Computing and Applied to Energy Decomposition for Demand-Side Management in a Smart Home
}

\author{
Yu-Chen Hu ${ }^{1}$, Yu-Hsiu Lin ${ }^{2, *}$ (i) and Harinahalli Lokesh Gururaj ${ }^{3}$ (D) \\ 1 Department of Computer Science and Information Management, Providence University, Taichung City 43301, \\ Taiwan; ychu@pu.edu.tw \\ 2 Graduate Institute of Automation Technology, National Taipei University of Technology, Taipei 106344, \\ Taiwan \\ 3 Department of Computer Science and Engineering, Vidyavardhaka College of Engineering, Mysuru 570002, \\ India; gururaj1711@vvce.ac.in \\ * Correspondence: yhlin@ntut.edu.tw; Tel.: +886-2-2771-2171 (ext. 4327)
}

check for updates

Citation: Hu, Y.-C.; Lin, Y.-H.; Gururaj, H.L. Partitional

Clustering-Hybridized Neuro-Fuzzy

Classification Evolved through

Parallel Evolutionary Computing and Applied to Energy Decomposition for Demand-Side Management in a Smart Home. Processes 2021, 9, 1539. https://doi.org/10.3390/pr9091539

Academic Editor: Shun-Hung Tsai

Received: 1 July 2021

Accepted: 26 August 2021

Published: 29 August 2021

Publisher's Note: MDPI stays neutral with regard to jurisdictional claims in published maps and institutional affiliations.

Copyright: (c) 2021 by the authors. Licensee MDPI, Basel, Switzerland. This article is an open access article distributed under the terms and conditions of the Creative Commons Attribution (CC BY) license (https:// creativecommons.org/licenses/by/ $4.0 /)$.

\begin{abstract}
The key advantage of smart meters over rotating-disc meters is their ability to transmit electric energy consumption data to power utilities' remote data centers. Besides enabling the automated collection of consumers' electric energy consumption data for billing purposes, data gathered by smart meters and analyzed through Artificial Intelligence (AI) make the realization of consumercentric use cases possible. A smart meter installed in a domestic sector of an electrical grid and used for the realization of consumer-centric use cases is located at the entry point of a household/building's electrical grid connection and can gather composite/circuit-level electric energy consumption data. However, it is not able to decompose its measured circuit-level electric energy consumption into appliance-level electric energy consumption. In this research, we present an AI model, a neuro-fuzzy classifier integrated with partitional clustering and metaheuristically optimized through parallelcomputing-accelerated evolutionary computing, that performs energy decomposition on smart meter data in residential demand-side management, where a publicly available UK-DALE (UK Domestic Appliance-Level Electricity) dataset is used to experimentally test the presented model to classify the On/Off status of monitored electrical appliances. As shown in this research, the presented AI model is effective at providing energy decomposition for domestic consumers. Further, energy decomposition can be provided for industrial as well as commercial consumers.
\end{abstract}

Keywords: Artificial Intelligence; energy decomposition; smart city; smart meter

\section{Introduction}

Nowadays, the electricity energy demand requested from downstream sectors of an electrical grid is continuously increasing. One way to meet the ever-increasing costs and demand for electricity energy that have led many organizations to find smart ways of monitoring, controlling, and saving electricity energy [1] is to make major/power-intensive electrical appliances in commercial, industrial, and residential buildings operate more efficiently in response to demand response schemes for Demand-Side Management (DSM) [2] (for the more intelligent use of electric power, i.e., the more efficient supply of electricity). Load monitoring technology is of great significance to DSM [3], where Energy Management Systems (EMSs) can contribute towards cutting costs while meeting demand requests [1]. DSM is the most promising technology that encourages consumers to optimize their electric energy consumption (to adjust their energy consumption behavior and usage habits [4]) such that: (1) the ever-increasing energy demand requested from downstream sectors of an electrical grid can be met; (2) the efficiency, reliability, and flexibility of the electrical grid can be improved; and (3) emissions of greenhouse gas such as carbon dioxide 
and chlorofluorocarbons can be abated. In an electrical grid to be upgraded as a smart grid, smart meters over rotating-disc meters have been developed and deployed as an advanced metering infrastructure for the automated collection of electric energy consumption data. A smart meter installed and located at the entry point of a building's electrical grid connection can acquire composite/circuit-level electric energy consumption, but it cannot identify the contributions of appliance-level electric energy consumption from its acquired composite/circuit-level electric energy consumption for load management in DSM. Appliance-level electric energy consumption on individual electrical appliances can be identified by plug-level power meters, called smart plugs, attached to relevant individual electrical appliances in a practical field of interest. However, the field is instrumented, which is significantly inconvenient for residents [5]. Moreover, monitoring relevant electrical appliances in an intrusive style is impractical as the investment costs, including costs for the installation and maintenance of infrastructure, are high [5]. Therefore, energy decomposition, so-called non-intrusive load monitoring [5-10], has been developed. Energy decomposition, as part of an EMS, is a process of decomposing composite/circuit-level electric energy consumption into appliance-level electric energy consumption through examining appliance-specific characteristics such as power consumption [6]; this comes with the advantage of requiring no additional plug-level power meters to be deployed for relevant individual electrical appliances while maintaining the investment costs for the energy management system, including its installation and annual maintenance costs, at a minimum. Energy decomposition can decompose composite/circuit-level electric energy consumption into appliance-level electric energy consumption based on feature data from relevant electrical appliances. Then, the time duration for which each of the electrical appliances was used can be further figured out, which is useful for actionable insights wherein the use of electrical appliances is scheduled in response to electricity pricing for DSM. Energy decomposition can be built upon an Artificial Intelligence (AI) methodology including metaheuristics [7-16], which ranges from machine learning to deep learning. For instance, in [9], the random forest, a meta estimator that fits a number of decision tree classifiers on various subsamples of training data and uses averaging/majority voting to improve predictive performance and control over-fitting, was used as the learning algorithm to determine which categories feature data belong to for energy decomposition. Energy decomposition involves the installation of a single minimal set of current and voltage sensors, such as a smart meter at the entry point of a building's electrical grid connection, to acquire the composite/circuit-level electric energy consumption. The composite/circuit-level electric energy consumption is processed by AI with signal processing technology. The composite/circuit-level energy consumption going into a building is decomposed into appliance-level energy consumption, in that it is capable of deducing what relevant electrical appliances for load management have been turned on/off, and the time duration for which each of the relevant electrical appliances was operated can be identified.

The AI methodology developed for energy decomposition in $[11,15,16]$ is based on a feedforward, multilayer ANN combined with particle swarm optimization. The main research direction here is to propose an energy decomposition approach based on a neurofuzzy classifier integrated with partitional clustering and metaheuristically optimized via parallel-computing-accelerated evolutionary computing. Neuro-fuzzy hybridization is a hybrid intelligent system that incorporates a fuzzy linguistic model of human-like reasoning with a connectionist structure of neurocomputing. The main strength of the hybridization is that it is a universal approximator with the ability to solicit interpretable IF-THEN fuzzy rules for solving problems of exactly mapping $x$ onto $y$. As a result, it is suitable for energy decomposition addressed as a classification problem. In this research, an energy decomposition approach based on a neuro-fuzzy classifier integrated with kMeans clustering and optimized metaheuristically by a parallel-computing-accelerated Genetic Algorithm (GA) is proposed and applied to identify the presence of relevant electrical appliances for load management. The neuro-fuzzy classifier with its adjustable 
parameters is initialized by k-Means clustering first. Then, the neuro-fuzzy classifier with its adjustable parameters coarsely determined by k-Means clustering is optimized via metaheuristics, GA, accelerated through parallel computing, wherein metaheuristics can fine tune the neuro-fuzzy classifier with its adjustable parameters as the classifier trained through gradient descent is easily fooled by local minima. In summary, in a new direction from the work done in $[11,15,16]$, we develop a neuro-fuzzy classifier, via neurofuzzy computing, further hybridized with partitional clustering (k-Means clustering) and metaheuristically evolved through parallel-computing-accelerated evolutionary computing (GA) for load classification in energy decomposition, in order to achieve optimal adjustable parameters of the hybridization where the evolutionary process for metaheuristically evolving the hybridization is sped up. Building upon connectionisms, one of the most promising bioinspired AI paradigms ever invented, neuro-fuzzy computing incorporates the human-like reasoning style of fuzzy systems, consisting of a set of fuzzy IF-THEN rules as a linguistic model with fuzzy sets, with the connectionist structure of neural networks, learning from data, as a hybrid intelligent system.

The organization of this research is as follows. The proposed energy decomposition approach is described in Section 2. Section 3 demonstrates it with a publicly available energy decomposition dataset. Lastly, our conclusions are presented in Section 4.

\section{Methodology}

The basic event-less energy decomposition process can be referenced in [7], where load recognition and load classification that utilizes AI, k-Means-clustering-hybridized and parallel-computing-accelerated GA-evolved neuro-fuzzy classification to recognize extracted electrical features for relevant electrical appliances was investigated.

In this research, evolutionary computing is used to metaheuristically optimize neurofuzzy classification initialized by k-Means clustering and trained without the use of any gradient information from mini-batch stochastic gradient descent, such as the Adam algorithm [17]. This methodology is described below.

\subsection{Neuro-Fuzzy Classification with $k$-Means Clustering}

Figure 1 shows the structure of neuro-fuzzy classification [18] conducted and integrated with k-Means clustering [19] in this research. A typical fuzzy system can be referenced in $[20,21]$. As shown in Figure 1, the fuzzifier transforms crisp input values from the present input into fuzzy sets. After the fuzzification process, fuzzified inputs are passed with created fuzzy IF-THEN rules to an inference engine that combines the created fuzzy IF-THEN rules using a fuzzy t-norm operator to derive each fuzzy IF-THEN rule's firing strength. A crisp output value corresponding to the present input is computed by a defuzzifier, after each fuzzy IF-THEN rule's firing strength is obtained. The crisp output is delivered to a decision-making unit to predict the class label—appliance class—of the present input-electrical features extracted as energy decomposition feature data from relevant electrical appliances for load management. The conducted neuro-fuzzy classification, via a neuro-fuzzy classifier, in this research can be represented as a three-layer feedforward network, as depicted in Figure 2. In this research, the development of the neuro-fuzzy classifier involves the following two steps. First, k-Means clustering $[19,22,23]$ embedding heuristic knowledge into the neuro-fuzzy classifier is used to construct the fuzzy IF-THEN rules of the neuro-fuzzy classifier from a training dataset. Each of the constructed fuzzy rules in the rule base is in charge of a partition of the feature space, where the considered electrical features are the universe of discourse. Second, a parallel GA is used to metaheuristically evolve the neuro-fuzzy classifier with the constructed fuzzy IF-THEN rules to a k-Means clustering-hybridized neuro-fuzzy classifier. 


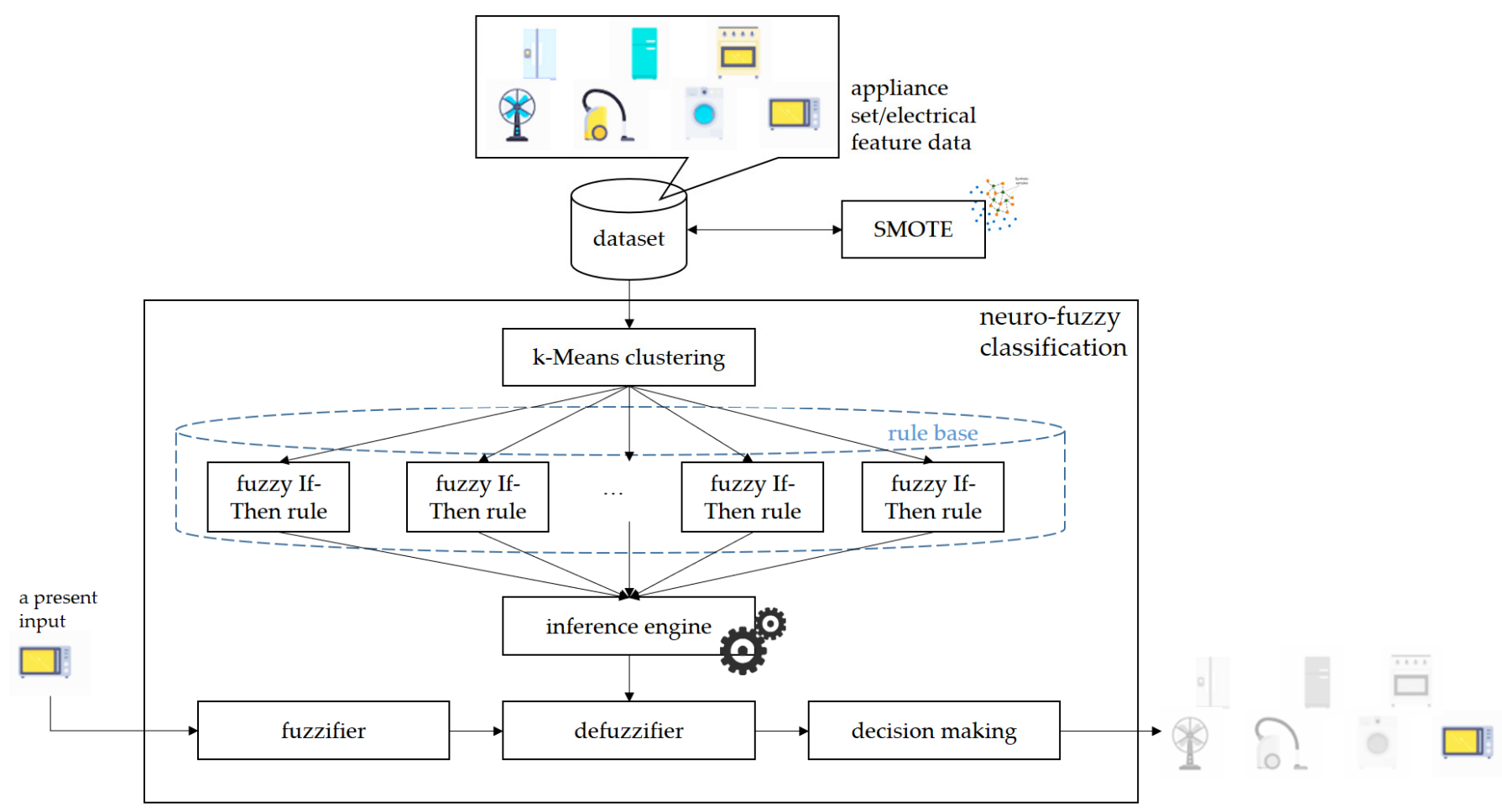

Figure 1. Structure of neuro-fuzzy classification integrated with k-Means clustering.

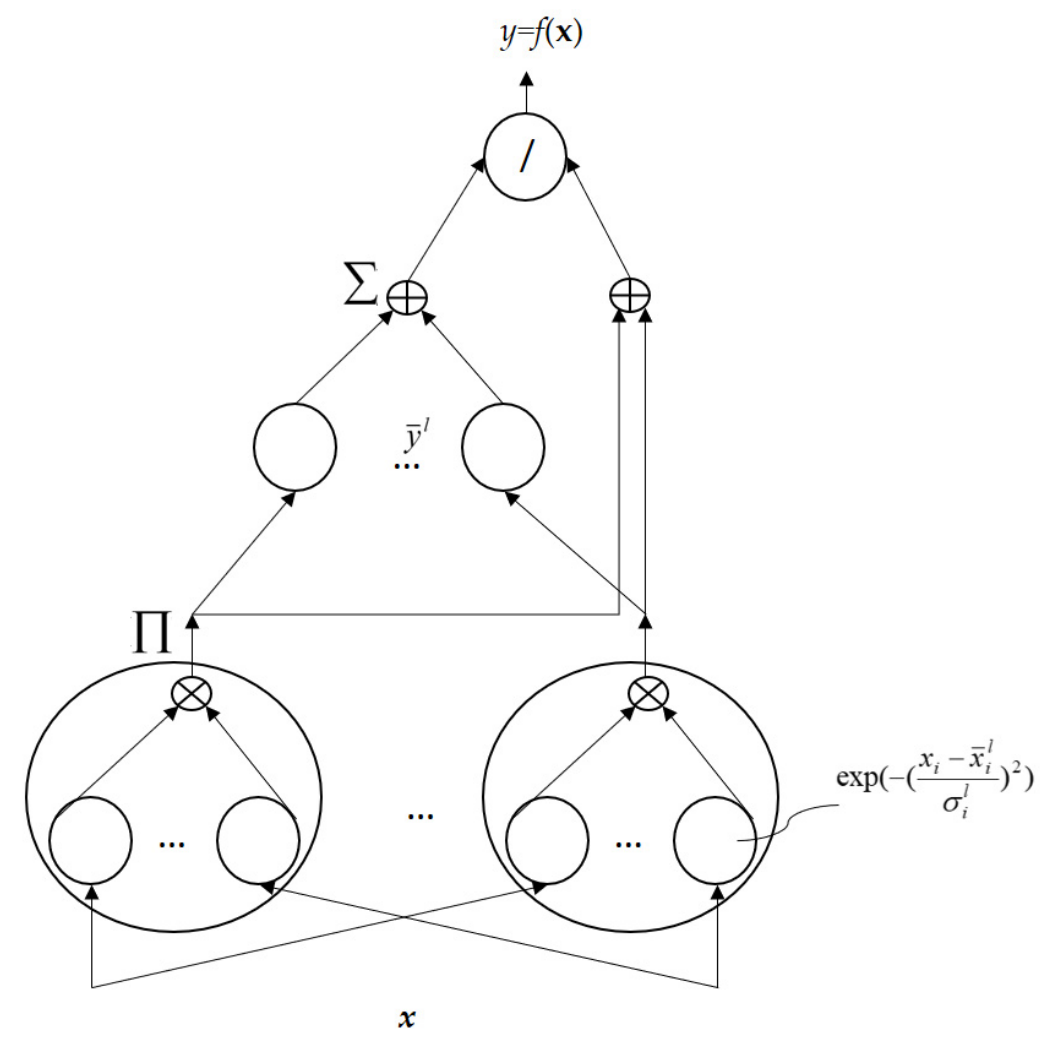

Figure 2. Network representation of the conducted neuro-fuzzy classification.

The neuro-fuzzy classifier is formulated as Equation (1). The singleton fuzzifier, product inference engine, and center average defuzzifier [18] are utilized. Also, its fuzzy rule base, made of a set of fuzzy IF-THEN rules, has the form of Equation (2). In Equation (2), $A_{i}{ }^{l}$ and $B^{l}$ are fuzzy sets in $U_{i} \subset R$ and $V \subset R$, respectively; $x=\left(x_{1}, x_{2}, \ldots, x_{i}, \ldots, x_{n}\right)^{\mathrm{T}}$ 
$\in U \subset R^{n}$ and $y \in V \subset R$ are the input and output linguistic variables of the neuro-fuzzy classifier, respectively; $M$ is the total number of fuzzy IF-THEN rules in the fuzzy rule

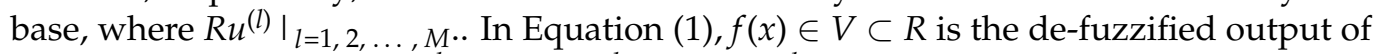
the neuro-fuzzy classifier; $\sigma_{i}^{l} \in(0, \infty), \bar{x}_{i}^{l} \in R$, and $\bar{y}^{l} \in R$ are real-valued parameters.

Each fuzzy set in the neuro-fuzzy classifier is characterized by a Gaussian membership function. In the neuro-fuzzy classifier, the Gaussian-style membership function was chosen instead of other styles of membership functions, such as triangular, trapezoidal, and generalized bell-shaped membership functions, for the following two reasons: First, the set of adjustable parameters to be trained/evolved is, in number, small. This is because each Gaussian membership function is characterized by two parameters-its center and spread parameters, $\bar{x}_{i}^{l}$ and $\sigma_{i}^{l}$, respectively. Second, the Gaussian-style membership function ensures that each fuzzy rule's firing strength is always nonzero. The center of fuzzy set $B^{l}$ of the neuro-fuzzy classifier is a singleton with a value of $\bar{y}^{l}$.

In this research, cluster means, $\left.\left\{\mu_{i}\right\}_{i=1}^{q}\right|_{q \leftarrow k}$, by k-Means clustering were used to allocate the center parameters of Gaussian membership functions (i.e., to center Gaussian membership functions) in the neuro-fuzzy classifier. Then, the spread parameters of the centered Gaussian membership functions in the neuro-fuzzy classifier are given as Equation (3) [24]. In Equation (3), the Gaussian membership functions are centered at $\left.\left\{\mu_{i}\right\}_{i=1}^{q}\right|_{q \leftarrow k}$ by $\mathrm{k}$ cluster centers/means. After the center and spread parameters of the neuro-fuzzy classifier are initialized, its $\bar{y}^{l}$ is also initialized from clustered data, where data with the same cluster mean are handled, with their class label, through majority voting (for multivariate variables/universes of discourse, redundant fuzzy rules can be eliminated).

$$
\begin{gathered}
f(x)=\frac{\sum_{l=1}^{M} \bar{y}^{l}\left[\prod_{i=1}^{n} \exp \left(-\left(\frac{x_{i}-\bar{x}_{i}^{l}}{\sigma_{i}^{l}}\right)^{2}\right)\right]}{\sum_{l=1}^{M}\left[\prod_{i=1}^{n} \exp \left(-\left(\frac{x_{i}-\bar{x}_{i}^{l}}{\sigma_{i}^{l}}\right)^{2}\right)\right]} \\
R u^{(l)}: \text { IF } x_{1} \text { is } A_{1}^{l} \text { and } \ldots \text { and } x_{n} \text { is } A_{n}^{l} \text {, THEN } y \text { is } B^{l} \\
\sigma=\max _{1 \leq i, j \leq q}\left\|\mu_{i}-\mu_{j}\right\| / \sqrt{2 q}
\end{gathered}
$$

Because the neuro-fuzzy classifier, as shown in Equation (1)/Figure 2, may be working with a class-imbalanced dataset, in this research, we use SMOTE (Synthetic Minority Oversampling Technique) [25] to generate synthetic data for minority classes and thus balance a class-imbalanced dataset before the classifier is constructed, fit, and used with a satisfactory level of classification performance for energy decomposition.

The adjustable parameters $\bar{x}_{i}^{l}, \sigma_{i}^{l}$, and $\bar{y}^{l}$ of the k-Means-clustering-hybridized neurofuzzy classifier above can be finetuned through gradient descent, where the updating rules to the three adjustable parameters of the k-Means-clustering-hybridized neuro-fuzzy classifier are provided in Equations (4)-(6). In Equation (4), $\eta$ is a tuning constant; $f$ and $y$ denote $f\left(x_{0}{ }^{p}\right)$ and $y_{0}{ }^{p}$, respectively; $a=\sum_{l=1}^{M} b^{l}$; and $b^{l}=\prod_{i=1}^{n} \exp \left(-\left(\frac{x_{i}-\bar{x}_{i}^{l}}{\sigma_{i}^{l}}\right)^{2}\right)$. If the k-Means-clustering-hybridized neuro-fuzzy classifier is satisfactory in that it is suitable for classifications from unseen data, the tuning process that updates the adjustable parameters using the next input-output data pair $\left.\left(x_{0}^{p+1}, y_{0}{ }^{p+1}\right)\right|_{p=p+1}$ ends; otherwise, it iteratively continues with $q=(q+1)$.

$$
\begin{gathered}
\bar{y}^{l}(q+1)=\bar{y}^{l}(q)-\eta \frac{f-y}{a} b^{l} \\
\bar{x}_{i}^{l}(q+1)=\bar{x}_{i}^{l}(q)-\eta \frac{f-y}{a}\left(\bar{y}^{l}(q)-f\right) b^{l} \frac{2\left(x_{0 i}^{p}-\bar{x}_{i}^{l}(q)\right)}{\sigma_{i}^{l 2}(q)} \\
\sigma_{i}^{l}(q+1)=\sigma_{i}^{l}(q)-\eta \frac{f-y}{a}\left(\bar{y}^{l}(q)-f\right) b^{l} \frac{2\left(x_{0 i}^{p}-\bar{x}_{i}^{l}(q)\right)^{2}}{\sigma_{i}^{l 3}(q)}
\end{gathered}
$$


The k-Means-clustering-hybridized neuro-fuzzy classifier is finetuned by Equations (4)-(6). Nevertheless, it is easily fooled by local minima. As a result, in our proposed classifier, GA is used to globally, metaheuristically search for the (quasi-)optimal adjustable parameters of the k-Means-clustering-hybridized neuro-fuzzy classifier. The GA and k-Means-clustering-hybridized neuro-fuzzy classifier is metaheuristically evolved in parallel as described in the following subsection.

\subsection{Parallel-Computing-Accelerated GA Used to Metaheuristically Optimize the k-Means-Clustering-Integrated Neuro-Fuzzy Classifier}

A GA can also be used to metaheuristically train (evolve) the k-Means-clusteringhybridized neuro-fuzzy classifier, a connectionism shown in Figure 2, without the use of any gradient information (an initial genetic search can be followed by a gradient method such as gradient descent, as described in the previous subsection). Parallel computing can be conducted and used to accelerate the GA as the GA involves extremely computationally intensive routines for function evaluations to evaluate a (very) large set of candidate solutions, i.e., chromosomes, for the (whole) algorithm to find the global (quasi-)optimal solution. The GA can be enhanced in terms of throughput and interactivity through parallel computing.

The first important step to using a GA to evolve the k-Means-clustering-hybridized neuro-fuzzy classifier is to encode the k-Means-clustering-hybridized neuro-fuzzy classifier into chromosomes (genotype representation). Each chromosome must then be decoded to a neuro-fuzzy classifier (phenotype representation) when each chromosome needs to be evaluated by a fitness function that guides the GA to find the global (quasi-)optimal solution. An illustration of the process of encoding the connectionism, shown in Figure 2, into a chromosome, a real-number string, for the GA as a real-number GA [26] is illustrated in Figure 3. The fitness function can be defined as $-E$, where $E$ is a cost function/loss function for which the k-Means-clustering-hybridized neuro-fuzzy classifier evolved by the GA seeks to minimize the error, such as Mean-Squared Error (MSE). With an initial population of randomly created chromosomes started for the GA, successive generations are constructed through evolution. During the evolutionary process, fitter chromosomes as higher-quality solutions are more likely to survive and participate in genetic operationsinvolving crossover and mutation operations-based on selection, mimicking Darwin's theory of evolution (natural selection). Selection is the driving force of the GA. The kMeans-clustering-hybridized neuro-fuzzy classifier metaheuristically trained (evolved) by the GA is not easily fooled by local minima.

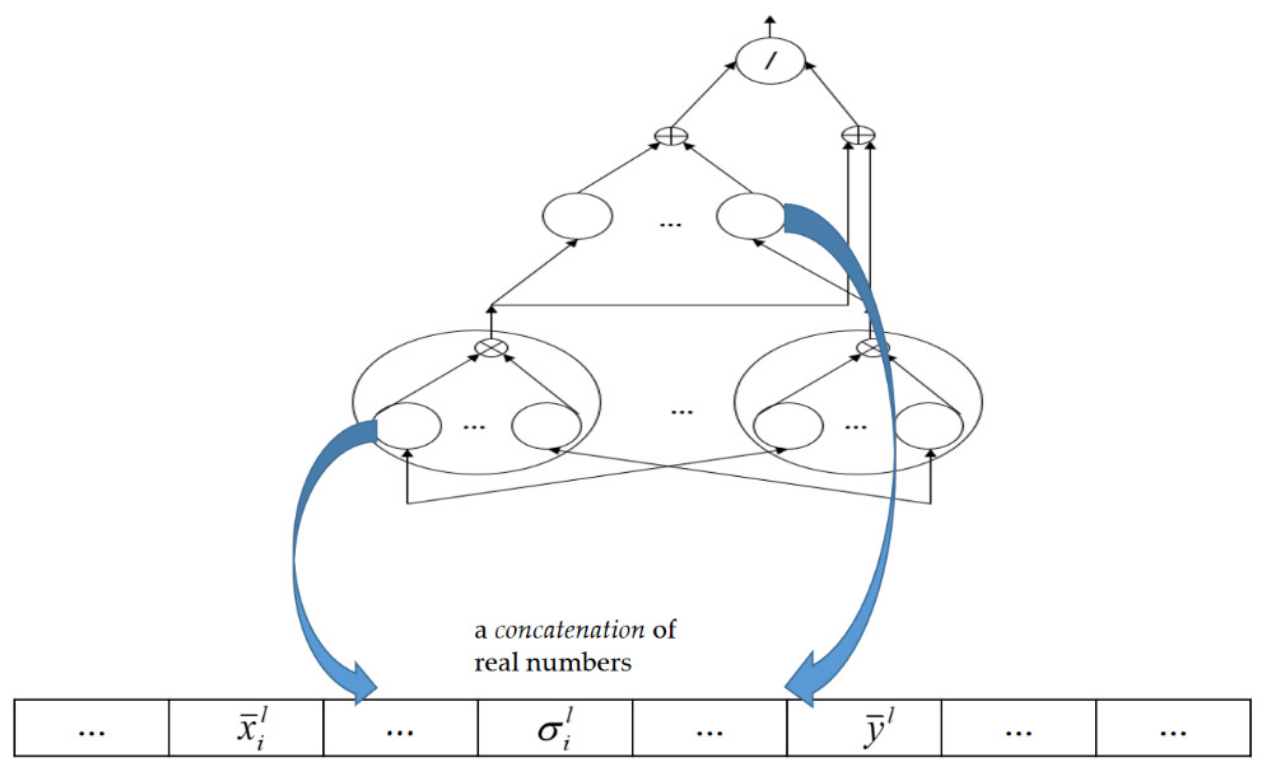

Figure 3. Illustration of the chromosomal design encoding the connectionism into a chromosome-a real-number string. 
Evolving the neuro-fuzzy classifier run over a massive training dataset to evaluate fitness during the evolutionary process is computationally intensive. It can be accelerated through parallel computing where the fitness evaluation during the evolutionary process is distributed among multiple processors, as multiple central processing units on a single computer, working as concurrent (parallel) workers. The GA is innately a parallel algorithm. Figure 4 illustrates a flowchart of the computational routines involved for fitness evaluation of the GA accelerated, in parallel, by multiple concurrent (parallel) workers. The loop executed for each neuro-fuzzy classifier run over electrical feature training data for fitness evaluation is also parallelized.
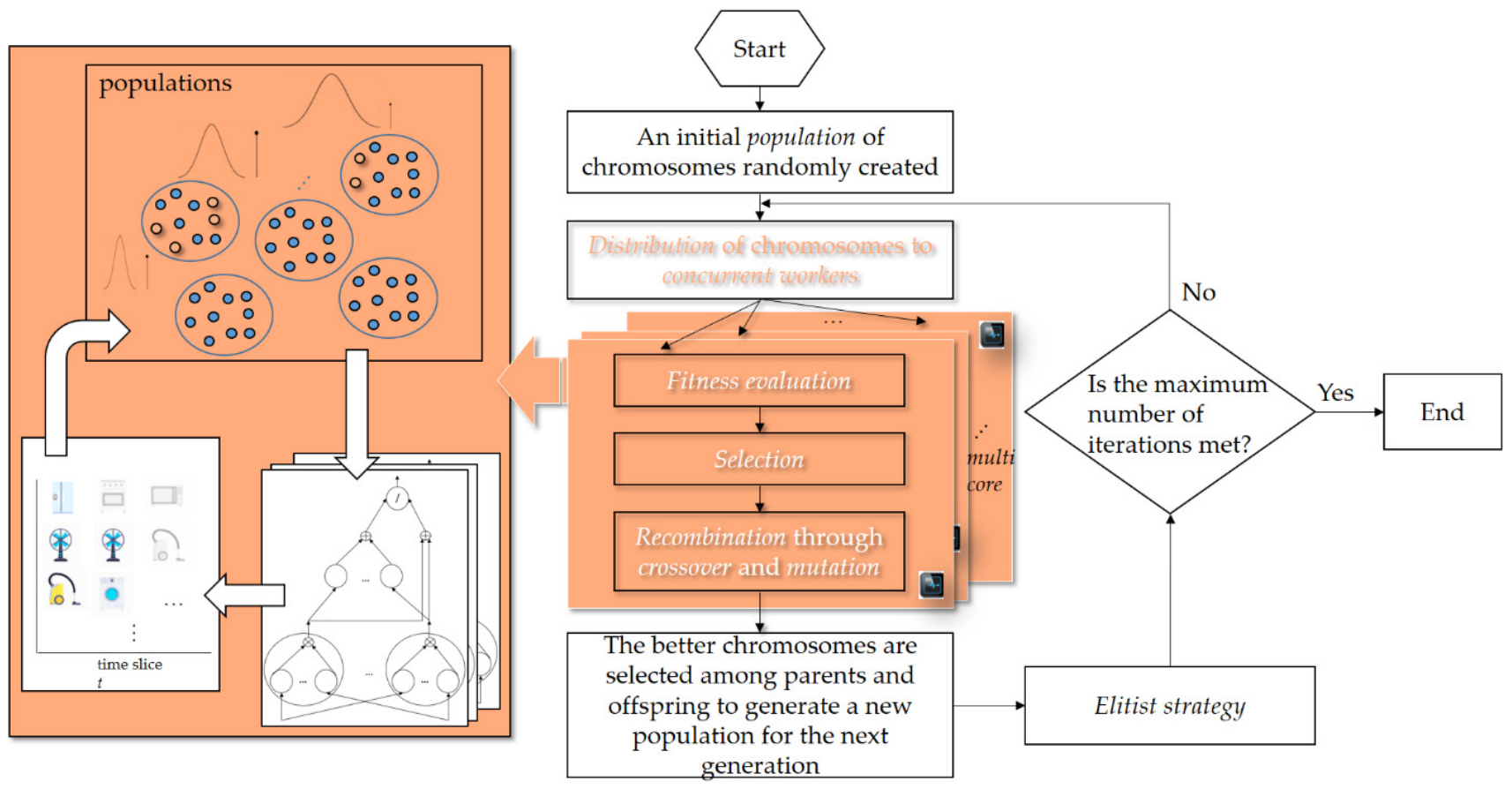

Figure 4. Flowchart of the computational routines involved for fitness evaluation of the parallel GA used to evolve the k-Means-clustering-hybridized neuro-fuzzy classifier for energy decomposition in this research.

In the GA accelerated via parallel computing, a selection strategy that breeds a new population/generation is adopted, where fitness scaling may be needed. Besides this, a crossover operator is used to combine selected chromosomes as parents producing offspring for the next population in the next generation of the evolutionary process. New offspring - new solutions - can be created to keep the population diversity high; these typically share many of the characteristics of the parents. Moreover, a mutation operator is utilized. Lastly, elite chromosomes are considered. In the GA, from generation to generation, the average fitness increases.

\section{Experimental Results}

In this research, an experiment was carried out to experimentally validate the effectiveness of the proposed energy decomposition approach, a k-Means-clustering-hybridized neuro-fuzzy classifier metaheuristically optimized by a parallel GA, that decomposes composite/circuit-level electric energy consumption into appliance-level electric energy consumption (which is addressed as an appliance classification problem) via energy decomposition. The UK-DALE dataset [27], conditioned in [7], was considered and used as a smart meter dataset. Figure 5 shows the considered historical power demand on Sunday 7 December 2014 in House 1, which was monitored and recorded in the UK-DALE dataset [27], conditioned in [7] and addressed in this research. The thin blue line shown in Figure 5a shows the total (composite) power demand in the mains; the stacked, filled, and colored blocks show the power demand by (1) the relevant individual electrical appli- 
ances and (2) devices monitored by all other submeters [27], treated together as one single individual in power absorptions and considered in energy decomposition. A base load, permanent load, and phantom load, which are shown in Figure $5 b$, were present in the house environment.

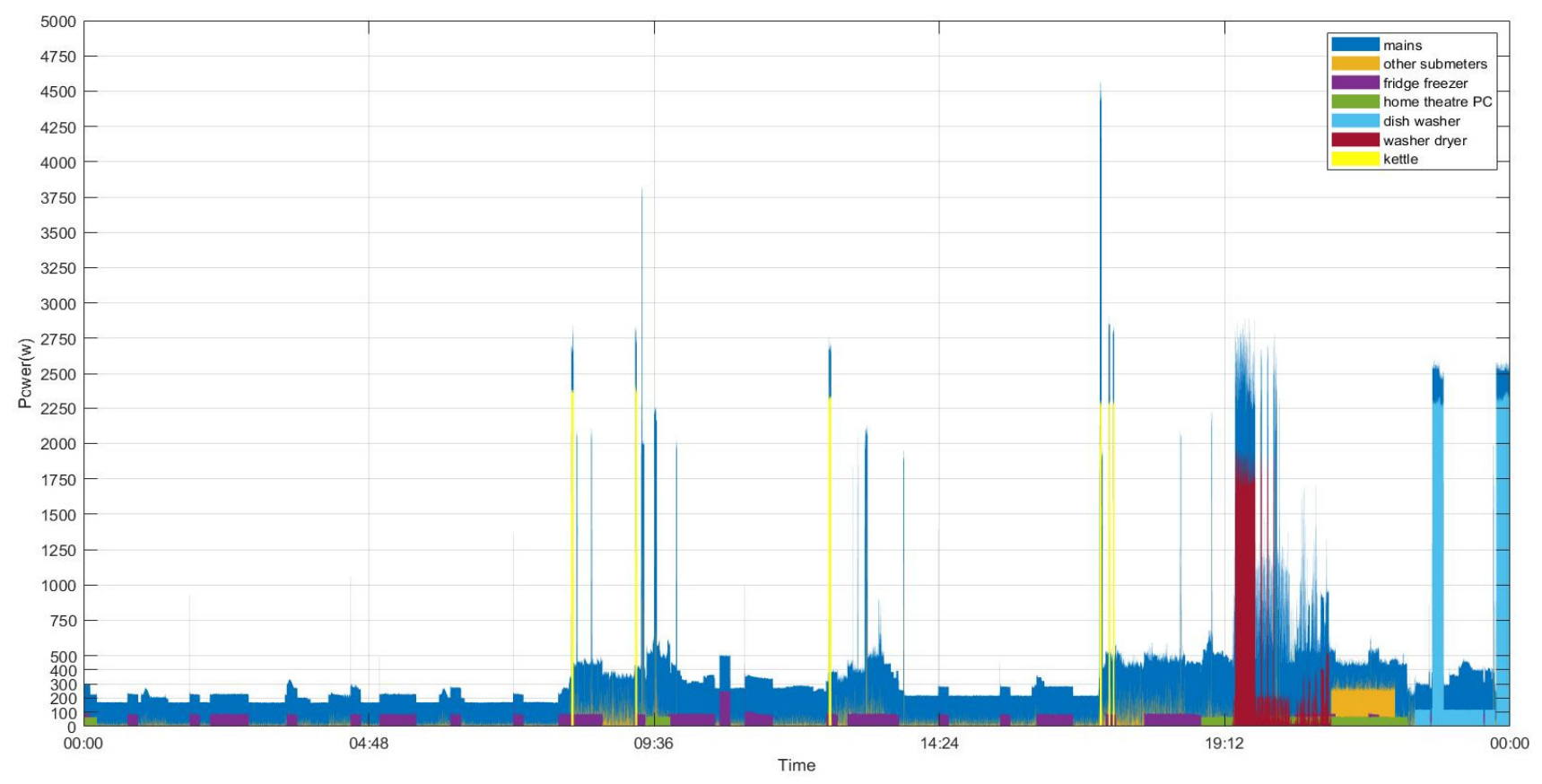

(a)

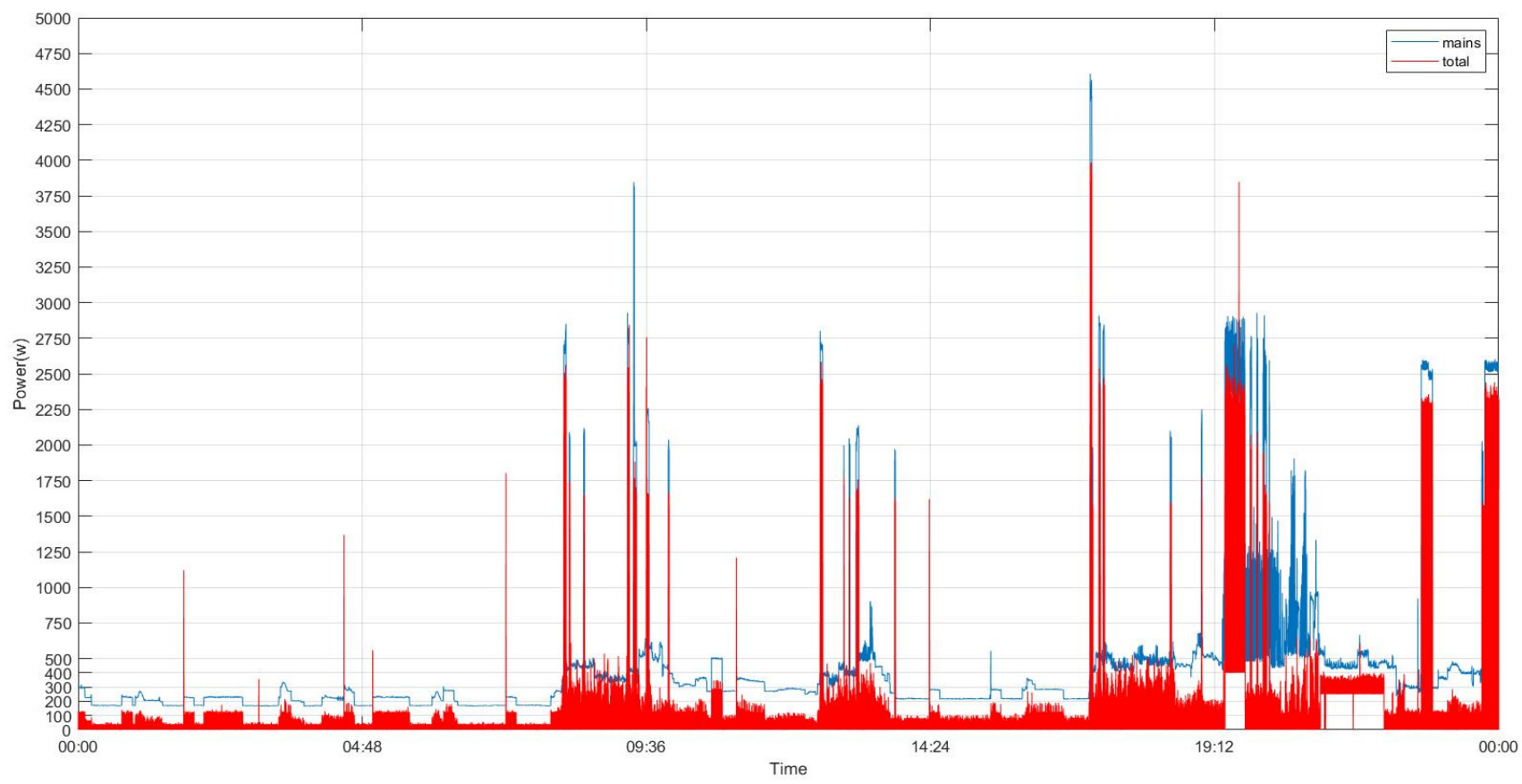

(b)

Figure 5. The considered historical power demand on Sunday 7 December 2014 in House 1, which was monitored and recorded in the UK-DALE dataset [27], conditioned in [7] and examined in this research: (a) the total (composite) power demand, to be decomposed into the individual power demand where base, permanent and phantom power demand existed, in the mains; (b) a base load, permanent load, and phantom load existed in the mains. This figure is from [7]. 
The relevant electrical appliances, including devices treated together as one single individual in power absorptions, are listed in Table 1, with their power demand shown in Figure 5a. In this experiment, in total, 989 composite/circuit-level power consumption data sampled from the historical power demand on Sunday 7 December 2014 (refer to Figure 5) and Thursday 4 December 2014 (which is shown in [7]) and prepared as an energy decomposition feature dataset were evaluated (decomposed/classified) using the proposed energy decomposition approach based on the k-Means-clustering-hybridized neuro-fuzzy classifier metaheuristically optimized by a parallel GA. A total of 15 load combinations (load classes) from the relevant electrical appliances in Table 1 were classified in the energy decomposition. The energy decomposition problem in [7] was treated as a multilabel classification problem, where theoretically there were a total of 4096 ( $\left.2^{12 \text { load classes }}\right)$ load combinations. Herein, the energy decomposition problem is considered as a multiclass classification problem. Table 2 tabulates the percentage of data for each class in the prepared energy decomposition feature dataset with or without the use of SMOTE.

Table 1. Relevant electrical appliances targeted for energy decomposition in this experiment/research.

\begin{tabular}{ccccc}
\hline \multirow{2}{*}{ Electrical Appliance } & \multicolumn{4}{c}{ Mean Power Consumption (Watts) } \\
\cline { 2 - 5 } & State 1 & State 2 & State 3 & State 4 \\
\hline fridge & 88.8 & 245.5 & - & - \\
\hline htpc & 68.5 & - & - & - \\
\hline washer/dryer & 182.2 & 1833.1 & - & - \\
\hline dishwasher & 116.0 & 2309.3 & - & - \\
\hline kettle & 2323.7 & - & 457.7 & 280.6 \\
\hline other submeters & 17.1 & 67.9 & &
\end{tabular}

Table 2. Checking the percentage of data for each class in the prepared energy decomposition feature dataset with or without the use of SMOTE.

\begin{tabular}{ccc}
\hline Class & \% (Without Use of SMOTE) & \% (With Use of SMOTE $\left.{ }^{\mathbf{1}}\right)^{(}$ \\
\hline 1 & 0.81 & 5.98 \\
\hline 2 & 2.33 & 6.58 \\
\hline 3 & 2.33 & 6.58 \\
\hline 4 & 50.25 & 18.80 \\
\hline 5 & 0.61 & 5.90 \\
\hline 6 & 0.91 & 6.02 \\
\hline 7 & 12.44 & 6.55 \\
\hline 8 & 0.10 & 0.04 \\
\hline 9 & 0.61 & 6.02 \\
\hline 10 & 21.44 & 9.16 \\
\hline 11 & 0.91 & 5.26 \\
\hline 12 & 1.52 & 4.35 \\
\hline 13 & 1.01 & 6.05 \\
\hline 14 & 2.12 & 6.13 \\
\hline 15 & 2.63 & 6.70 \\
\hline
\end{tabular}

${ }^{1}$ For the majority class, undersampling can be performed by a clustering method that generates centroids from data.

As seen on the left-hand side in Table 2, the prepared energy decomposition feature dataset is a highly class-imbalanced dataset that needs to be handled before it is addressed 
(learned) by the proposed energy decomposition approach. The widely adopted technique for handling a highly class-imbalanced dataset is "resampling", which consists of removing existing data from the majority classes (which is called undersampling) and/or generating more data for the minority classes (which is called oversampling). In this experiment, SMOTE (Synthetic Minority Oversampling Technique) [25], which synthesizes data for minority classes to balance a class-imbalanced dataset, was used to balance the prepared energy decomposition feature dataset. The dataset after SMOTE was balanced, as seen on the right-hand side in Table 2, and a balanced energy decomposition feature dataset was thus obtained. SMOTE synthesizes data based on the data that already exist in the minority classes, which works by (1) randomly choosing data (data samples) from the minority classes, (2) identifying their nearest neighbors prespecified by an integer, and then (3) generating synthetic data for them with respect to their identified neighbors (two neighbors were considered in this experiment).

In this experiment, the proposed energy decomposition approach, a k-Means-clusteringhybridized neuro-fuzzy classifier metaheuristically optimized by a parallel GA, was implemented in MATLAB ${ }^{\circledR}$ and run on an Acer Predator G3-710 Intel ${ }^{\circledR}$ Core $^{\mathrm{TM}}$ i7-6700 CPU (3.40 GHz) (RAM: $16 \mathrm{~GB})$ personal computer where parallel computing was performed based on four available concurrent (parallel) workers. For the k-Means-clusteringhybridized neuro-fuzzy classifier (Figure 2), $\mathrm{k}$ was set to 15 (the total number of load classes was 15). The total number of universes of discourse for the input of the k-Means-clusteringhybridized neuro-fuzzy classifier was one. The universe of discourse was divided into 15 partitions $(\mathrm{k}=15)$ by 15 Gaussian membership functions. Thus, the total number of fuzzy IF-THEN rules was also 15.

Table 3 shows the cluster centers found by the k-Means clustering and used to center the 15 Gaussian membership functions, where their spread parameter was initialized according to Equation (3). For the GA (refer to Figure 4) used to metaheuristically optimize the k-Means-clustering-hybridized neuro-fuzzy classifier and accelerated via parallel computing, a random initial population was created with a uniform distribution in the initial population range by k-Means clustering (the initial population range was $[-10.0,10.0]$ ). Here, $\bar{x}_{i}^{l}, \sigma_{i}^{l}$, and $\bar{y}^{l}$ were bound by $\left[\left.\left\{\mu_{i}\right\}_{i=1}^{q}\right|_{q \leftarrow k}-\alpha \cdot \sigma,\left.\left\{\mu_{i}\right\}_{i=1}^{q}\right|_{q \leftarrow k}+\beta \cdot \sigma\right],[1, \gamma \cdot \sigma]$, and $\left[1,\left.q\right|_{q \leftarrow k}\right]$, respectively; $\alpha, \beta$, and $\gamma$ were constants falling in $(0,1]$. In this experiment, $\alpha, \beta$, and $\gamma$ were set to $0.1,0.1$, and 1 , respectively. The parameters specified for the GA are as follows: The population size was 350 . The initial population was created at random in real-number strings, where the total length of each chromosome was 45 (a total of $15_{\text {membership functions }} \times 2$ parameters per function $\times 1$ input $+15_{\text {rules }}$ need to be evolved). The stochastic uniform selection [28] was adopted, where fitness scaling was considered. The scattered crossover operator [28] was used to recombine selected chromosomes as parents producing offspring for the next population of the evolutionary process (a user-defined crossover operator such as affine crossover, which is one of the arithmetic crossover operators, can be specified); the crossover fraction of the population to be evolved from generation to generation was 0.75 . The Gaussian mutation operator [28] was utilized; the mutation rate was 0.01 . Elite chromosomes, the top $(0.05 \times$ the population size $)$ chromosomes that are guaranteed to survive from their current population to the next population, were considered. The maximum number of generations was 200. Lastly, the fitness function was defined as $-E$, where $E$ denotes MSE (the objective of the GA is to minimize MSE by the neuro-fuzzy classifier).

Table 3. Cluster centers found by the k-Means clustering and used to center the 15 Gaussian membership functions in this experiment. 
Figure 6 shows the evolutionary trajectory obtained by the GA. During the evolutionary process, the loop executed for each neuro-fuzzy classifier (phenotype representation) run over the training data for the fitness evaluation was also parallelized by 'parfor' executing for-loop iterations in parallel on workers in a parallel pool [29]. Figure 7 shows the training trajectory of the k-Means-clustering-hybridized neuro-fuzzy classifier trained through gradient descent. As shown in Figure 7, the MSE obtained was 4.29, and the obtained overall classification rate was $75.68 \%$. Referring to Figure 6, the best MSE obtained was 2.95 , where the obtained overall classification rate was $82.43 \%$. The obtained overall classification rate thus improved, against $75.68 \%$, by $6.75 \%$. As shown in Figure 4, evolutionary computing can be used as an initial genetic search and followed with gradient descent. In this experiment, the GA was used as an initial genetic search and followed with gradient descent for the neuro-fuzzy classifier, as shown in Figure 8. As shown in Figure 8, which presents the training trajectory of the neuro-fuzzy classifier trained through gradient descent after the GA completed, the MSE obtained was 2.68 and the overall classification rate was $86.49 \%$. The obtained overall classification rate was thus improved, against $75.68 \%$, by $10.81 \%$. Table 4 summarizes the overall classification rates obtained in this experiment. As shown in Table 4, the proposed energy decomposition approach, the kMeans-clustering-hybridized neuro-fuzzy classifier metaheuristically optimized by parallel GA as an initial genetic search and then trained through gradient descent, outperformed the other examined approaches.

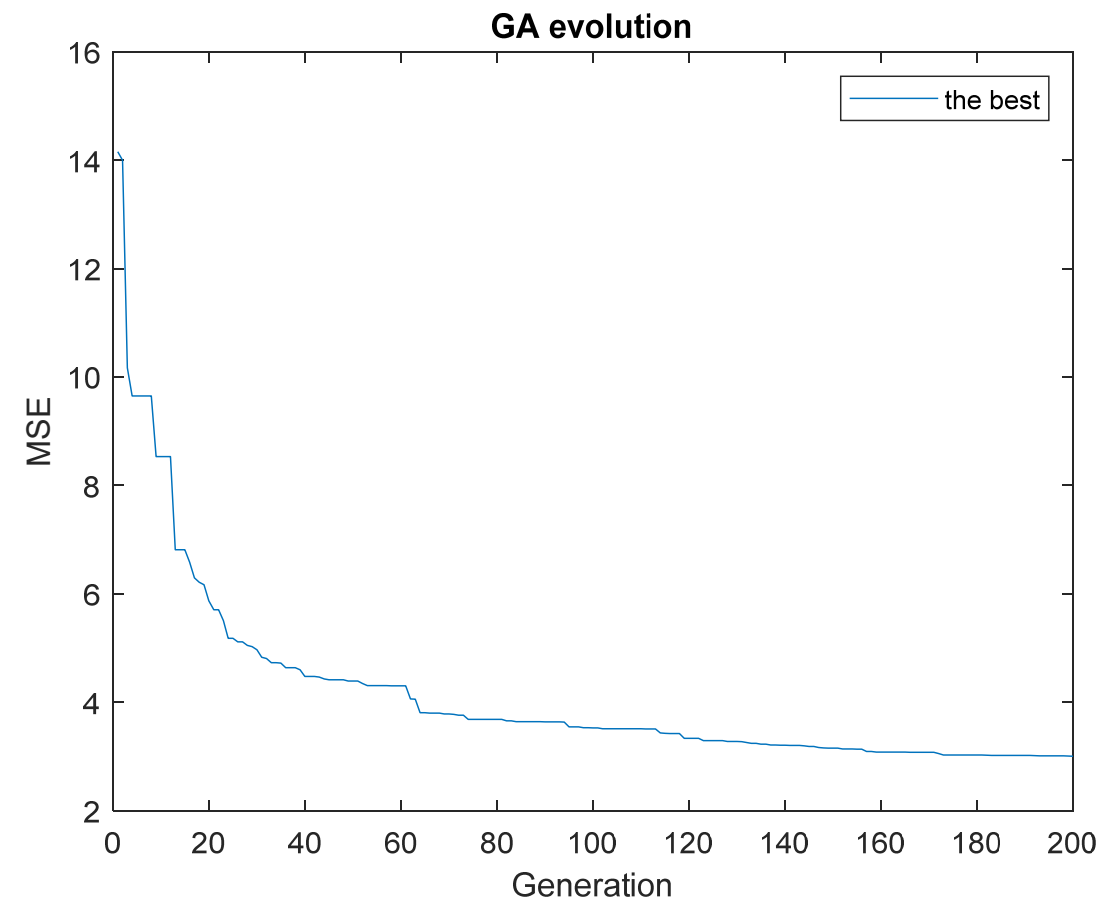

Figure 6. Evolution trajectory obtained by the GA specified, executed, and accelerated for the optimization of the k-Means-clustering-hybridized neuro-fuzzy classifier in this experiment. The GA was configured in terms of (population size, crossover fraction, mutation rate) at $(350,0.75,0.01)$. The GA determined the fitness value, MSE, where the lower the error, the higher the fitness. 


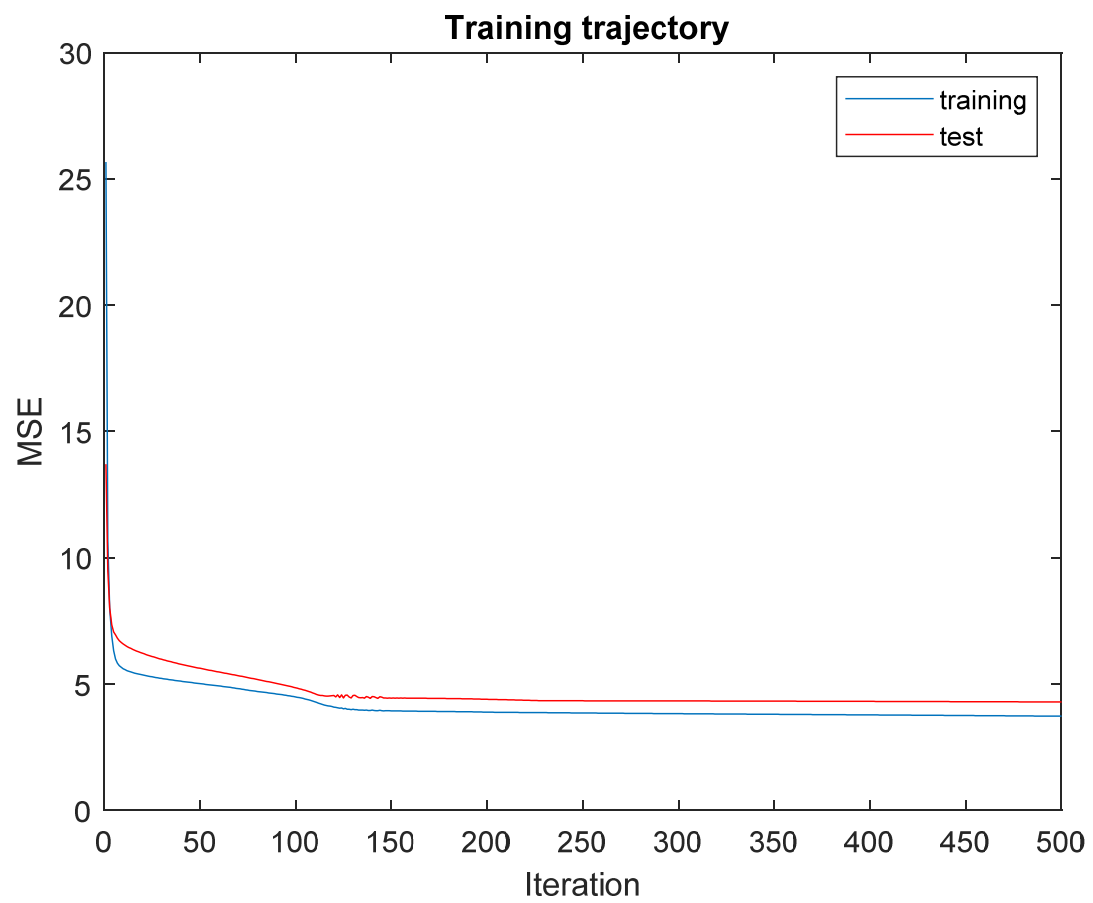

Figure 7. Training trajectory of the k-Means-clustering-hybridized neuro-fuzzy classifier trained through gradient descent.

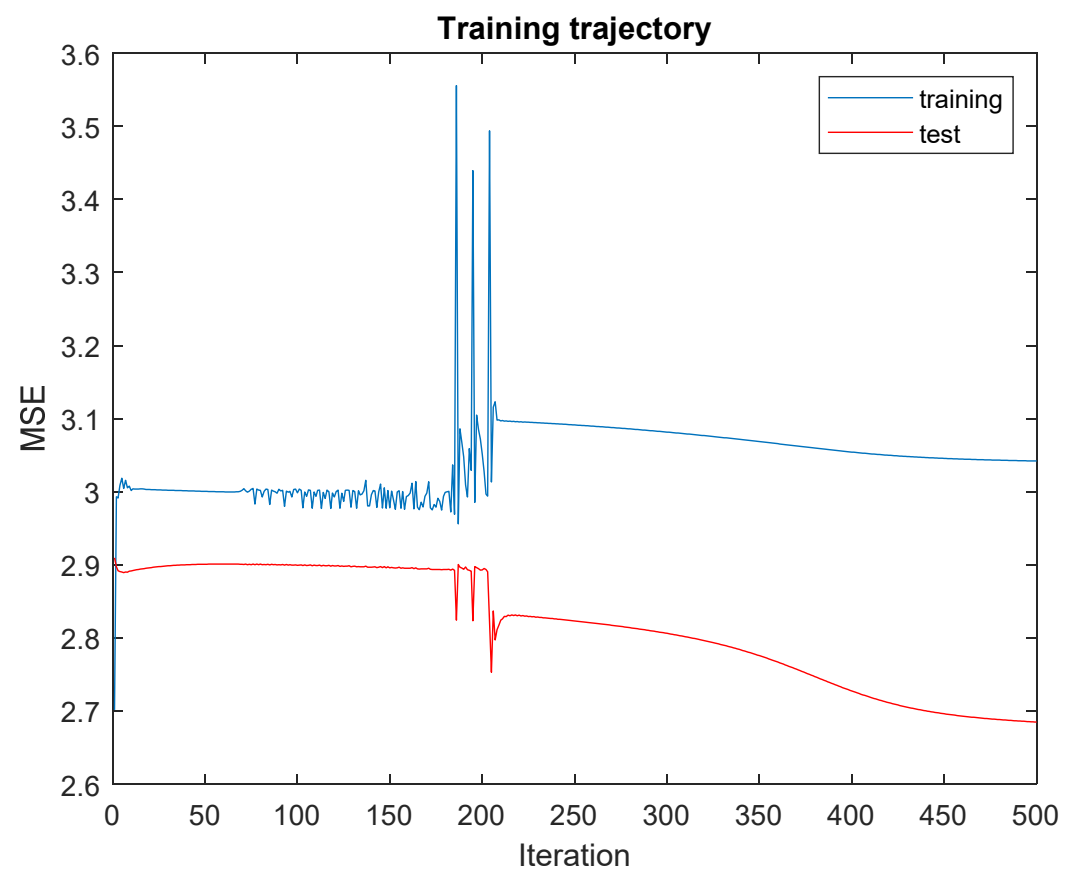

Figure 8. Training trajectory of the neuro-fuzzy classifier trained through gradient descent after the initial genetic search was completed. 
Table 4. Overall classification rates obtained in this experiment.

\begin{tabular}{cccc}
\hline & \multicolumn{3}{c}{ Metrics } \\
\hline $\begin{array}{c}\text { Optimization Technique Incorporated with } \\
\text { the k-Means-Clustering-Hybridized } \\
\text { Neuro-Fuzzy Classifier }\end{array}$ & MSE & Overall Classification Rate (\%) & Improvement (\%) \\
\hline gradient descent ${ }^{1}$ & 4.29 & 75.68 & - \\
\hline parallel GA & 2.95 & 82.43 & 6.75 \\
\hline parallel GA + gradient descent & 2.68 & 86.49 & 10.81 \\
\hline
\end{tabular}

In [7], a feedforward, multilayer ANN-based energy decomposition method was used to perform energy decomposition on the UK-DALE dataset. Also, in [12], two different types of ANNs, Autoencoder and Long Short-Term Memory (LSTM), were used. A comparison among the neuro-fuzzy hybridization-based energy decomposition approach proposed in this research, an ANN-based energy decomposition method inspired by $[7,11,15,16]$, and the two different types of ANNs-the Autoencoder and the LSTMapplied in [12] on the UK-DALE dataset for energy decomposition is shown in Table 5. As shown in Table 5, the proposed energy decomposition approach, reporting an averaged accuracy score of $86.49 \%$, outperformed the LSTM, reporting an averaged accuracy score of $70.33 \%$. The proposed energy decomposition approach versus the Autoencoder was capable of discriminating the targeted electrical appliances with an acceptable level of classification accuracy.

Table 5. Comparison among the proposed energy decomposition approach, an ANN-based energy decomposition method, the Autoencoder, and the LSTM for energy decomposition.

\begin{tabular}{|c|c|c|c|c|}
\hline & $\begin{array}{c}\text { The Neuro-Fuzzy } \\
\text { Hybridization-Based } \\
\text { Energy } \\
\text { Decomposition } \\
\text { Approach Proposed in } \\
\text { this Research }\end{array}$ & $\begin{array}{c}\text { Feedforward, } \\
\text { Multilayer } \\
\text { ANN-Based Energy } \\
\text { Decomposition } \\
\text { Inspired by } \\
{[7,11,15,16]}\end{array}$ & $\begin{array}{c}\text { Autoencoder Used in } \\
{[12]}\end{array}$ & LSTM Used in [12] \\
\hline $\begin{array}{c}\text { averaged accuracy } \\
\text { score }(\%)\end{array}$ & 86.49 & 61.27 & 94.00 & 70.33 \\
\hline
\end{tabular}

To conclude, as demonstrated and reported in this experiment, the proposed energy decomposition approach, the k-Means-clustering-hybridized neuro-fuzzy classifier metaheuristically optimized by the GA via parallel computing, can be applied to energy decomposition feature data, namely, the considered and conditioned publicly available UK-DALE data (as smart meter data), with a satisfactory level of classification performance regarding energy decomposition in (residential) load management/DSM.

\section{Conclusions and Future Work}

Smart meter data, comprising composite/circuit-level electric energy consumption data captured at the entry point of a household/building's electrical grid connection, can be further analyzed and used for the realization of useful consumer-centric use cases. DSM is one such useful consumer-centric use cases. Energy decomposition can be used to decompose composite/circuit-level electric energy consumption into appliance-level electric energy consumption, based on feature data from relevant electrical appliances. The time duration for which each of the electrical appliances was used can be further figured out, which is useful for actionable insights in which the electrical appliances are scheduled in response to electricity pricing for DSM. The AI methodology developed for energy decomposition in the literature is based on a feedforward, multilayer ANN integrated with a metaheuristic. The main research direction in this research was to propose an energy decomposition approach based on neuro-fuzzy hybridization. This was 
further hybridized with partitional clustering and optimized metaheuristically via parallelcomputing-accelerated evolutionary computing. Neuro-fuzzy hybridization is a hybrid intelligent system that incorporates a fuzzy linguistic model of human-like reasoning with a connectionist structure of neurocomputing. The main strength of the hybridization is that it is a universal approximator with the ability to solicit interpretable IF-THEN fuzzy rules to solve problems of exactly mapping $x$ onto $y$. Therefore, we proposed a new energy decomposition approach, a neuro-fuzzy classifier integrated with partitional clustering and metaheuristically optimized through parallel-computing-accelerated evolutionary computing, to decompose composite/circuit-level electric energy consumption into appliance-level electric energy consumption for energy decomposition in load management/DSM, as the presence of relevant electrical appliances is identified. The proposed energy decomposition approach involves the following two steps: First, k-Means clustering is used to initialize the neuro-fuzzy classifier with its adjustable parameters. Second, metaheuristics, namely, a GA accelerated by parallel computing, is conducted and used to evolve the neuro-fuzzy classifier initialized by k-Means clustering. The neuro-fuzzy classifier with its adjustable parameters is metaheuristically optimized by GA because the neuro-fuzzy classifier trained through gradient descent is easily fooled by local minima. The initial GA can then be followed by gradient descent. As shown in this research, the proposed energy decomposition approach can be applied to smart meter data, such as the publicly available UK-DALE data, with a satisfactory level of classification performance for energy decomposition in (residential) load management/DSM. In the future, the proposed energy decomposition approach will be evaluated on other datasets, like BLUED (Building-Level fUlly-labeled dataset for Electricity Disaggregation), REDD (Reference Energy Disaggregation Data set), and PLAID (Plug-Level Appliance Identification Dataset). Developed for services provided for domestic consumers, energy decomposition can conduct predictive analytics methodology to provide services such as fault detection and diagnosis of rotating machinery for industrial and commercial consumers.

Author Contributions: Y.-C.H. conceived, designed, and performed the experiment and contributed related experimental tools/materials to analyze the experimental data. Y.-H.L. validated the experimental results. Y.-H.L. and H.L.G. conceived, designed, and performed the experiments. Y.-H.L. and Y.-C.H. wrote the paper. All authors have read and agreed to the published version of the manuscript.

Funding: The Ministry of Science and Technology, Taiwan, under grant nos. MOST 109-2221-E027-121-MY2, MOST 110-3116-F-006-001-, and MOST 110-3116-F-027-001-, for which Y.-H.L. is the principal investigator or co-principal investigator, partly supported this research.

Institutional Review Board Statement: Not applicable.

Informed Consent Statement: Not applicable.

Data Availability Statement: Not applicable.

Acknowledgments: The authors would like to sincerely thank the reviewers and editor for their valuable comments and suggestions on this research.

Conflicts of Interest: The authors declare no conflict of interest.

\section{References}

1. Al-Ali, A.R.; Zualkernan, I.A.; Rashid, M.; Gupta, R.; Alikarar, M. A smart home energy management system using IoT and big data analytics approach. IEEE Trans. Consum. Electron. 2017, 63, 426-434. [CrossRef]

2. Samuel, O.; Javaid, S.; Javaid, N.; Ahmed, S.H.; Afzal, M.K.; Ishmanov, F. An efficient power scheduling in smart homes using Jaya based optimization with Time-of-Use and Critical Peak Pricing schemes. Energies 2018, 11, 3155. [CrossRef]

3. Zhao, B.; Stankovic, L.; Stankovic, V. On a training-less solution for non-intrusive appliance load monitoring using graph signal processing. IEEE Access 2016, 4, 1784-1799. [CrossRef]

4. Iglesias, F.; Palensky, P.; Cantos, S.; Kupzog, F. Demand side management for stand-alone hybrid power systems based on load identification. Energies 2012, 5, 4517-4532. [CrossRef]

5. Wu, S.; Lo, K.L. Non-intrusive monitoring algorithm for resident loads with similar electrical characteristic. Processes 2020, 8 , 1385. [CrossRef] 
6. Li, D.; Dick, S. Non-intrusive load monitoring using multi-label classification methods. Electr. Eng. 2021, 103, 607-619. [CrossRef]

7. Hu, Y.-C.; Lin, Y.-H.; Lin, C.-H. Artificial intelligence, accelerated in parallel computing and applied to nonintrusive appliance load monitoring for residential demand-side management in a smart grid: A comparative study. Appl. Sci. 2020, 10, 8114. [CrossRef]

8. Wu, X.; Jiao, D.; Du, Y. Automatic implementation of a self-adaption non-intrusive load monitoring method based on the convolutional neural network. Processes 2020, 8, 704. [CrossRef]

9. $\mathrm{Wu}, \mathrm{X}$;; Gao, Y.; Jiao, D. Multi-label classification based on random forest algorithm for non-intrusive load monitoring system. Processes 2019, 7, 337. [CrossRef]

10. Çavdar, İ.H.; Faryad, V. New design of a supervised energy disaggregation model based on the deep neural network for a smart grid. Energies 2019, 12, 1217. [CrossRef]

11. Lin, Y.-H.; Hu, Y.-C. Electrical energy management based on a hybrid artificial neural network-particle swarm optimizationintegrated two-stage non-intrusive load monitoring process in smart homes. Processes 2018, 6, 236. [CrossRef]

12. Kelly, J.; Knottenbelt, W. Neural NILM: Deep neural networks applied to energy disaggregation. In Proceedings of the 2nd ACM International Conference on Embedded Systems for Energy-Efficient Built Environments (ACM BuildSys'15), Seoul, Korea, 4-5 November 2015; pp. 55-64.

13. Guillén-García, E.; Morales-Velazquez, L.; Zorita-Lamadrid, A.L.; Duque-Perez, O.; Osornio-Rios, R.A.; de Jesús Romero-Troncoso, R. Identification of the electrical load by $\mathrm{C}$-means from non-intrusive monitoring of electrical signals in non-residential buildings. Int. J. Electr. Power Energy Syst. 2019, 104, 21-28. [CrossRef]

14. Zheng, Z.; Chen, H.; Luo, X. A Supervised Event-Based Non-Intrusive Load Monitoring for Non-Linear Appliances. Sustainability 2018, 10, 1001. [CrossRef]

15. Chang, H.H.; Chen, K.L.; Tsai, Y.P.; Lee, W.J. A new measurement method for power signatures of non-intrusive demand monitoring and load identification. IEEE Trans. Ind. Appl. 2012, 48, 764-771. [CrossRef]

16. Chang, H.-H. Non-intrusive demand monitoring and load identification for energy management systems based on transient feature analyses. Energies 2012, 5, 4569-4589. [CrossRef]

17. Kingma, D.P.; Ba, J. Adam: A method for stochastic optimization. In Proceedings of the 3rd International Conference for Learning Representations (ICLR2015), San Diego, CA, USA, 7-9 May 2015; pp. 1-13.

18. Wang, L.X. Design of Fuzzy Systems from Input-Output Data. A Course in Fuzzy Systems and Control, International ed.; Pearson Education Taiwan Ltd.: Taipei, Taiwan, 2005; pp. 168-172.

19. Wang, L.X. Miscellaneous Topics. A Course in Fuzzy Systems and Control, International ed.; Pearson Education Taiwan Ltd.: Taipei, Taiwan, 2005; pp. 343-347.

20. Tayyaba, S.; Ashraf, M.W.; Alquthami, T.; Ahmad, Z.; Manzoor, S. Fuzzy-based approach using IoT devices for smart home to assist blind people for navigation. Sensors 2020, 20, 3674. [CrossRef]

21. Ain, Q.-u.; Iqbal, S.; Khan, S.A.; Malik, A.W.; Ahmad, I.; Javaid, N. IoT operating system based fuzzy inference system for home energy management system in smart buildings. Sensors 2018, 18, 2802. [CrossRef]

22. Zhu, X.; Jin, X.; Jia, D.; Sun, N.; Wang, P. Application of data mining in an intelligent early warning system for rock bursts. Processes 2019, 7, 55. [CrossRef]

23. Zhou, M.; Zhang, Q.; Liu, Y.; Sun, X.; Cai, Y.; Pan, H. An integration method using kernel principal component analysis and cascade support vector data description for pipeline leak detection with multiple operating modes. Processes $2019,7,648$. [CrossRef]

24. Kumar, S. Neural Networks: A Classroom Approach, International ed.; McGraw-Hill Education (Asis): New Delhi, India, 2005; pp. 304-329.

25. Chawla, N.V.; Bowyer, K.W.; Hall, L.O.; Kegelmeyer, W.P. SMOTE: Synthetic minority over-sampling technique. J. Artif. Intell. Res. 2002, 16, 321-357. [CrossRef]

26. Lin, C.-J.; Sie, T.-Y.; Chu, W.-L.; Yau, H.-T.; Ding, C.-H. Tracking control of pneumatic artificial muscle-activated robot arm based on sliding-mode control. Actuators 2021, 10, 66. [CrossRef]

27. Kelly, J.; Knottenbelt, W. The UK-DALE dataset, domestic appliance-level electricity demand and whole-house demand from five UK homes. Sci. Data 2015, 2, 150007. [CrossRef] [PubMed]

28. Genetic Algorithm Options-MATLAB \& Simulink-MathWorks. Available online: https://www.mathworks.com/help/gads/ genetic-algorithm-options.html (accessed on 29 April 2021).

29. Parfor-Execute for-Loop Iterations in Parallel on Workers-MathWorks. Available online: https://www.mathworks.com/help/ parallel-computing/parfor.html (accessed on 23 April 2021). 